\title{
Distinguishing between HII regions and planetary nebulae with Hi-GAL, WISE, MIPSGAL, and GLIMPSE ${ }^{\star \star \star}$
}

\author{
L. D. Anderson ${ }^{1, \star \star \star}$, A. Zavagno ${ }^{1}$, M. J. Barlow ${ }^{2}$, P. García-Lario ${ }^{3}$, and A. Noriega-Crespo ${ }^{4}$ \\ ${ }^{1}$ Laboratoire d'Astrophysique de Marseille (UMR 6110 CNRS \& Université de Provence), 38 rue F. Joliot-Curie, \\ 13388 Marseille Cedex 13, France \\ e-mail: Loren. Anderson@mail. wvu. edu \\ 2 Department of Physics and Astronomy, University College London, Gower Street, London WC1E 6BT, UK \\ 3 Herschel Science Centre, European Space Astronomy Centre, ESA, Madrid, Spain \\ ${ }^{4}$ SPITZER Science Center, California Institute of Technology, Pasadena, CA 91125, USA
}

Received 6 July 2011 / Accepted 13 October 2011

\begin{abstract}
Context. H II regions and planetary nebulae (PNe) both emit at radio and infrared (IR) wavelengths, and angularly small $\mathrm{H}$ II regions can be mistaken for PNe. This problem of classification is most severe for H II regions in an early evolutionary stage, those that are extremely distant, or those that are both young and distant. Previous work has shown that H II regions and PNe can be separated based on their infrared colors.

Aims. Using data from the Herschel Hi-GAL survey, as well as WISE and the Spitzer MIPSGAL and GLIMPSE surveys, we wish to establish characteristic IR colors that can be used to distinguish between $\mathrm{H}$ II regions and PNe.

Methods. We perform aperture photometry measurements for a sample of $126 \mathrm{H}$ II regions and $43 \mathrm{PNe}$ at wavelengths from $8.0 \mu \mathrm{m}$ to $500 \mu \mathrm{m}$.

Results. We find that $\mathrm{H}$ II regions and PNe have distinct IR colors. The most robust discriminating color criteria are $\left[F_{12} / F_{8}\right]<0.3$, $\left[F_{160} / F_{12}\right]>1.3$, and $\left[F_{160} / F_{24}\right]>0.8$ (or alternately $\left[F_{160} / F_{22}\right]>0.8$ ), where the brackets indicate the $\log$ of the flux ratio. All three of these criteria are individually satisfied by over $98 \%$ of our sample of $\mathrm{H}$ II regions and by $\sim 10 \%$ of our sample of PNe. Combinations of these colors are more robust in separating the two populations; for example all H II regions and no PNe satisfy $\left[F_{12} / F_{8}\right]<0.4$ and $\left[F_{160} / F_{22}\right]>0.8$. When applied to objects of unknown classification, these criteria prove useful in separating the two populations. The dispersion in color is relatively small for $\mathrm{H}$ II regions; this suggests that any evolution in these colors with time for $\mathrm{H}$ II regions must be relatively modest. The spectral energy distributions (SEDs) of H II regions can be separated into "warm" and "cold" components. The "cold" component is well-fit by a grey-body of temperature $25 \mathrm{~K}$. The SEDs of nearly two-thirds of our sample of H II regions peak at $160 \mu \mathrm{m}$ and one third peak at $70 \mu \mathrm{m}$. For PNe, 67\% of the SEDs peak at $70 \mu \mathrm{m}, 23 \%$ peak at either $22 \mu \mathrm{m}$ or $24 \mu \mathrm{m}$, and $9 \%$ (two sources) peak at $160 \mu \mathrm{m}$.
\end{abstract}

Key words. infrared: ISM - stars: formation - dust, extinction - planetary nebulae: general - HII regions

\section{Introduction}

Despite their different origins, HII regions and planetary nebulae (PNe) have similar characteristics at infrared (IR) and radio wavelengths ${ }^{1}$. The dust associated with $\mathrm{HII}$ regions and $\mathrm{PNe}$ is responsible for their emission at mid-IR (MIR) to farIR (FIR) wavelengths. For H II regions this emission is from dust within the $\mathrm{HII}$ region itself and from the photodissociation region (PDR), which is the interface between the ionized gas and the interstellar medium (see recent results from, e.g.

\footnotetext{
* Herschel is an ESA space observatory with science instruments provided by European-led Principal Investigator consortia and with important participation from NASA.

$\star \star$ Tables 1 and 2 are available at the CDS via anonymous ftp to cdsarc.u-strasbg.fr (130.79.128.5) or via http://cdsarc.u-strasbg.fr/viz-bin/qcat?]/A+A/537/A1

$\star \star \star$ Current Address: Physics Department, West Virginia University, Morgantown, WV 26506, USA

1 Technically PNe and H II regions are both "H II regions" in that they contain ionized plasma. Throughout we exclusively refer to "H II regions" as the ionized zones surrounding massive stars, and their associated dust and gas.
}

Deharveng et al. 2010; Anderson et al. 2010; Rodón et al. 2010; Anderson 2011). The IR emission from the PDRs of H II regions comes from neutral material possibly collected during the expansion of the H II region. For PNe, the IR emission is from the dusty layers of material shed during their creation.

Recently, there has been extensive work on the IR photometric properties of large samples of PNe. PNe have been studied using the IRAC instrument (Fazio et al. 2004) on Spitzer by Hora et al. (2004); Cohen et al. (2007); Kwok et al. (2008); Phillips \& Ramos-Larios (2008); Zhang \& Kwok (2009); Cohen et al. (2011) and also with the MIPS instrument (Rieke et al. 2004) on Spitzer by Chu et al. (2009); Zhang \& Kwok (2009); Phillips \& Marquez-Lugo (2011). Most pertinent for the present study are the results of Cohen et al. $(2007,2011)$, who showed that PNe should be well-separated from both ultra-compact (UC) H II regions and also more evolved H II regions in IRAC MIR colorcolor diagrams. These results, however, were based on simulated $\mathrm{H}$ II region colors and it remains to be seen if they can be replicated in real measurements.

$\mathrm{H}$ II regions and $\mathrm{PNe}$ also emit at radio wavelengths. In both cases, the central star(s) produce ultra-violet radiation that ionizes the surrounding medium. This ionized gas emits radio 
continuum radiation via thermal Bremsstrahlung (free-free). The radio continuum emission from $\mathrm{H}$ II regions is extremely bright; it has been known for some time that most bright continuum sources in the Galactic plane are H II regions. For example, 75\% of the bright radio continuum sources in Westerhout (1958) are $\mathrm{HII}$ regions and none are PNe. While not as bright, the radio continuum emission from $\mathrm{PNe}$ has also been detected for numerous objects (Milne \& Aller 1975; Zijlstra et al. 1989; Aaquist \& Kwok 1990; Condon \& Kaplan 1998). The stars powering H II regions emit considerably more ionizing flux than those powering $\mathrm{PNe}$, and therefore the radio emission from $\mathrm{HII}$ regions can be detected at larger distances.

Numerous authors have used data from the IRAS point source catalog to establish criteria for identifying H II regions and PNe. Wood \& Churchwell (1989) found that UC H II regions, which are generally young and small in angular size, have characteristic colors of $\left[F_{25} / F_{12}\right] \geq 0.57$ and $\left[F_{60} / F_{12}\right] \geq 1.30$, where $F_{\lambda}$ is the IRAS flux at wavelength $\lambda$ and the brackets indicate a base ten logarithm. Hughes \& MacLeod (1989) found that $\mathrm{H}$ II regions have characteristic colors $\left[F_{25} / F_{12}\right] \geq 0.4$ and $\left[F_{60} / F_{12}\right] \geq 0.25$ while PNe have $\left[F_{25} / F_{12}\right] \geq 0.4$ and $\left[F_{60} / F_{12}\right] \leq 0.25$. Using different IRAS flux bands, Pottasch et al. (1988) found PNe generally have colors $\left[F_{25} / F_{12}\right] \geq 0.46$ and $\left[F_{60} / F_{25}\right] \leq 0.52$. These studies demonstrate the power of FIR criteria for locating and discriminating between $\mathrm{H}$ II regions and PNe.

Candidate H II regions and PNe can in many cases be distinguished based on visual inspection of their IR emission (e.g. Cohen et al. 2007, 2011). H II regions tend to have more extended emission that PNe, and they show less symmetry. The central stars of PNe are frequently detected at near-IR wavelengths, and this has been used in the "Red MSX Survey" (RMS) survey (Urquhart et al. 2008) to distinguish between the two types of object. While a visual inspection of sources is sufficient in many cases, there are two cases in which visual inspection fails: young H II regions that are not yet extended at IR wavelengths, and evolved H II regions at extreme distances from the Sun. In both cases, the resolution of current MIR surveys does not allow one to distinguish between $\mathrm{H}$ II regions and PNe. The problem is the most severe in the case of young, distant H II regions. Visual inspection of course also fails for studies of extragalactic $\mathrm{H}$ II regions and PNe where the resolution is insufficient.

The recently completed Green Bank Telescope HII Region Discovery Survey (GBT HRDS; Bania et al. 2010; Anderson et al. 2011) illustrates the problem of separating H II regions from PNe. The HRDS sources were selected on the basis of spatially coincident radio and MIR emission. While this criterion easily distinguishes thermally emitting sources (e.g. H II regions and $\mathrm{PNe}$ ) from non-thermally emitting sources (e.g. supernova remnants and active galactic nuclei), it is not sufficient to distinguish between $\mathrm{HII}$ regions and $\mathrm{PNe}$. The number of $\mathrm{PNe}$ detected in the HRDS is likely low (Bania et al. 2010), although Anderson et al. (2011) mention a number of cases where the source classification is unclear. The HRDS sources are the most distant H II regions yet detected (Anderson, in prep.). Their small angular size may prevent the detection of more diffuse emission, making visual classification unreliable.

The goal of the present work is to establish IR criteria that can be used to distinguish between $\mathrm{HII}$ regions and PNe. These criteria can then be applied to samples of objects of unknown classification, which could greatly increase the number of known H II regions and PNe. The Herschel Hi-GAL survey (Molinari et al. 2010), together with WISE (Wright et al. 2010), Spitzer MIPSGAL (Carey et al. 2009), and Spitzer GLIMPSE
(Benjamin et al. 2003; Churchwell et al. 2009) allow us to revisit IR criteria for separating the two classes of objects, at much higher resolution and sensitivity compared to IRAS.

\section{Data}

\subsection{Herschel Hi-GAL}

The on-going Hi-GAL survey uses the PACS (Poglitsch et al. 2010) and SPIRE (Griffin et al. 2010) instruments of the Herschel Space Observatory to map the Galactic plane over the zone $+70^{\circ} \geq l \geq-70^{\circ},|b| \leq 1^{\circ}$, and was recently awarded time to map $\sim 240$ square degrees in the outer Galaxy as well. Hi-GAL contains photometry bands centered at $70 \mu \mathrm{m}$ and $160 \mu \mathrm{m}$ with PACS, and $250 \mu \mathrm{m}, 350 \mu \mathrm{m}$, and $500 \mu \mathrm{m}$ with SPIRE. The spatial resolutions of these bands are 6.7", 11", 18", 25", and 37", respectively. The point source sensitivities of Hi-GAL measured in a complex field are $0.5,4.1,4.1,3.2$, and $2.5 \mathrm{Jy} /$ beam for the $70 \mu \mathrm{m}, 160 \mu \mathrm{m}, 250 \mu \mathrm{m}, 350 \mu \mathrm{m}$, and $500 \mu \mathrm{m}$ bands while those in a less complex field are $0.06,0.9,0.7,0.7$, and $0.8 \mathrm{Jy} /$ beam (Molinari et al. 2010). The FIR coverage of Hi-GAL traces the emission of dust; the $70 \mu \mathrm{m} \mathrm{Hi-GAL}$ band is sensitive to warm $(\sim 100 \mathrm{~K})$ dust while the longer wavelength bands are more sensitive to colder $(\sim 10-30) \mathrm{K}$ dust.

\subsection{WISE}

The Wide-field Infrared Survey Explorer (WISE) mapped the entire sky in four IR bands: $3.4 \mu \mathrm{m}, 4.6 \mu \mathrm{m}, 12 \mu \mathrm{m}$, and $22 \mu \mathrm{m}$. The data used here are from the preliminary data release of 14 April 2011, which cover 57\% of the sky. Along the Galactic plane, this coverage extends approximately from $60^{\circ} \geq \ell \geq$ $-70^{\circ}$ and $240^{\circ} \geq \ell \geq 120^{\circ}$. The spatial resolutions in the four bands are 6 .' $^{\prime} 1,6$. $^{\prime \prime} 4,66^{\prime \prime} 5$, and $12^{\prime \prime}$ and the sensitivities are $0.08 \mathrm{mJy}, 0.11 \mathrm{mJy}, 1 \mathrm{mJy}$, and $6 \mathrm{mJy}$, respectively.

Here we use only the WISE $12 \mu \mathrm{m}$ and $22 \mu \mathrm{m}$ bands. The WISE $12 \mu \mathrm{m}$ band should trace similar dust emission components as that of the IRAC $8.0 \mu \mathrm{m}$ band. The $12 \mu \mathrm{m}$ band, however, is significantly broader than that of the IRAC $8.0 \mu \mathrm{m}$ band; within this broad band are PAH features at $11.2 \mu \mathrm{m}, 12.7 \mu \mathrm{m}$, and $16.4 \mu \mathrm{m}$ (see Tielens 2008). The PAH features at $7.7 \mu \mathrm{m}$ and $8.6 \mu \mathrm{m}$ also fall within the bandpass although at diminished sensitivity. There are also nebular emission lines that may be strong in evolved PNe. The $22 \mu \mathrm{m}$ band should trace the same dust emission components as the $24 \mu \mathrm{m}$ MIPSGAL band (see below).

The WISE $22 \mu \mathrm{m}$ band saturates for point sources at $12.4 \mathrm{Jy}$ (see WISE explanatory supplement ${ }^{2}$ ) which is six times higher than that for MIPSGAL, 2 Jy (Carey et al. 2008). We thus expect the WISE $22 \mu \mathrm{m}$ band to be less affected by problems caused by saturation compared to the MIPSGAL $24 \mu \mathrm{m}$ band. The WISE $12 \mu \mathrm{m}$ band saturates for point sources at $0.9 \mathrm{Jy}$.

The WISE image data have units of DN and we use the DNto-Jy conversion factors of $2.9045 \times 10^{-6}$ and $5.2269 \times 10^{-5}$ for the $12 \mu \mathrm{m}$ and $22 \mu \mathrm{m}$ bands, respectively (see WISE explanatory supplement ${ }^{2}$ ).

\subsection{Spitzer MIPSGAL}

The Spitzer MIPSGAL survey mapped the Galactic plane over $+60^{\circ} \geq l \geq-60^{\circ},|b| \leq 1^{\circ}$ using the MIPS instrument.

\footnotetext{
2 http://wise2.ipac. caltech.edu/docs/release/prelim/ expsup/wise_prelrel_toc.html
} 
MIPSGAL used two of the three MIPS photometric bands centered at $24 \mu \mathrm{m}$ and $70 \mu \mathrm{m}$. Here we use only data from the $24 \mu \mathrm{m}$ band, which has a spatial resolution of $6^{\prime \prime}$. MIPSGAL saturates at $\sim 1700 \mathrm{MJy} \mathrm{sr}^{-1}$ Carey et al. (2008), and strong saturation is present for many star-forming regions and for some PNe (see Sect. 5). Unlike the IRAC bands, the MIPS $24 \mu \mathrm{m}$ data have minimal correction for extended source fluxes (Cohen 2009) and we apply no such correction when computing fluxes.

For H II regions, the $24 \mu \mathrm{m}$ emission is detected from two distinct zones: along the PDR and also spatially coincident with the ionized gas. These components contribute roughly equally to the $24 \mu \mathrm{m}$ flux of H II regions (Deharveng et al. 2010; Anderson 2011). The emission coincident with the ionized gas likely comes from very small grains (VSGs) out of thermal equilibrium. The emission from the PDR is probably a combination of emission from VSGs and from a larger grain population whose temperature is closer to $25 \mathrm{~K}$. For PNe, the emission at $24 \mu \mathrm{m}$ also likely contains contributions from dust at a range of temperatures, but there may also be nebular emission lines that contribute to the emission in this band.

\subsection{Spitzer GLIMPSE}

The Spitzer Galactic Legacy Infrared Mid-Plane Survey Extraordinaire (GLIMPSE) mapped the Galactic plane over the same Galactic zone as MIPSGAL using the IRAC instrument. GLIMPSE contains four MIR bands at $3.6 \mu \mathrm{m}, 4.5 \mu \mathrm{m}, 5.8 \mu \mathrm{m}$, and $8.0 \mu \mathrm{m}$ at resolutions of $\sim 2^{\prime \prime}$. Here we use only the $8.0 \mu \mathrm{m}$ band because, relative to the other GLIMPSE bands, this band contains fewer stars and more intense diffuse emission. This makes source fluxes easier to measure and decreases errors in the aperture photometry. In addition to being sensitive to warm dust, the $8.0 \mu \mathrm{m}$ filter contains polycyclic aromatic hydrocarbon (PAH) emission from PAH features at $7.7 \mu \mathrm{m}$ and $8.6 \mu \mathrm{m}$ (see Tielens 2008). PAH molecules fluoresce when excited by far$\mathrm{UV}$ photons and thus emit very strongly in $\mathrm{H}$ II regions (see ISO spectra in Peeters et al. 2002a,b). PAH emission is moderately strong for C-rich PNe and is weak or absent for O-rich PNe (Volk \& Kwok 2003; Bernard-Salas 2006).

Scattering within the focal plane causes an increase in the measured flux of extended sources with the IRAC instrument, an effect that is wavelength dependent. To correct for this effect, we follow the Spitzer recommendations ${ }^{3}$ and apply an aperture correction to the $8.0 \mu \mathrm{m}$ fluxes, based on the size of the aperture. This correction lowers the measured flux values by a maximum of $35 \%$ for apertures $\gtrsim 50^{\prime \prime}$.

\subsection{IRAS}

The IRAS satellite mapped $98 \%$ of the sky at 12, 25, 60, and $100 \mu \mathrm{m}$. In Sect. 5.2.1 we use reprocessed IRAS data known as IRIS (Miville-Deschênes \& Lagache 2005) to compare against WISE $12 \mu \mathrm{m}$ fluxes. IRIS improves on the original IRAS data by correcting for calibration issues, the zero level, and striping problems. The angular resolution of the IRIS data is $3 .^{\prime} 8 \pm 0.2$, $3.8 \pm 0.2,4.0 \pm 0.2$, and $4.3 \pm 0.2$ for the $12,25,60$, and $100 \mu \mathrm{m}$ bands, respectively.

\footnotetext{
${ }^{3}$ See http://web.ipac.caltech.edu/staff/jarrett/irac/ calibration/index.html
}

\section{Source samples}

We require that all $\mathrm{H}$ II regions and $\mathrm{PNe}$ fall within the boundaries of all the IR surveys used here. At the time of this writing, the Hi-GAL coverage is the most restrictive. It includes longitude coverage from $346^{\circ} .5>\ell>290^{\circ}$, and $3.5>\ell>-4.5$, in addition to a few other more isolated fields. We restrict our samples to the above stated ranges in the southern Galactic plane and about the Galactic center.

\subsection{HII regions}

Our goal in creating the $\mathrm{H} \mathrm{II}$ region sample is to have $\mathrm{H}$ II regions spanning a range of evolutionary stages. We make no attempt to create an H II region sample with angular sizes matching that of the PN sample. While many H II regions could not be confused with $\mathrm{PNe}$ due to their large angular sizes, if they were located $20 \mathrm{kpc}$ from the Sun the situation would be less clear. By including H II regions spanning a range of physical sizes, we can account for the two situations where an H II region may be confused with a PN: young compact H II regions in an early evolutionary stage and more evolved $\mathrm{H}$ II regions at extreme distances from the Sun.

We compile our sample of H II regions from (a) the HRDS (Anderson et al. 2011), (b) the catalog of H II regions detected in recombination line emission prior to the HRDS described in Anderson et al. (2011) (henceforth identified as the "known" sample), and (c) sources classified as $\mathrm{H}$ II regions in the rms survey. The angular sizes of the HRDS targets are typically $\lesssim 2^{\prime}$, but many are extremely distant (Anderson, in prep.). The regions known prior to the HRDS consist mainly of those sources in the recombination line surveys of Caswell \& Haynes (1987) and Lockman (1989) for the Galactic zone here studied, although there are also contributions from Lockman et al. (1996), and Sewilo et al. (2004). The RMS survey contains objects detected as point sources in the MSX survey (Egan et al. 2003), excluding the zone within $10^{\circ}$ of the Galactic center. Since they are unresolved by MSX, which has an angular resolution of 18". 3 (Price et al. 2001), the MSX targets are small in angular size. The MSX sources used here that were classified as H II regions by the RMS survey all have detected CO emission (Urquhart et al. 2007b) and radio continuum emission (Urquhart et al. 2007a), which together solidify their classification as H II regions. As with the $\mathrm{PNe}$, we only include $\mathrm{HII}$ regions in relatively uncomplicated fields.

Our H II region sample contains 126 sources: 21 from the HRDS, 49 from the known sample, and 56 from the RMS survey. This sample of HII regions contains older, evolved regions in the "known" sample, younger H II regions from the RMS survey (many of which are in the ultra-compact phase, as indicated by their compact radio continuum emission measured by Urquhart et al. 2007a), and the HRDS sources, which likely span a range of evolutionary stages (Anderson, in prep.).

Example HII region images are shown in Fig. 1, which shows data from $8.0 \mu \mathrm{m}$ to $500 \mu \mathrm{m}$. At $8.0 \mu \mathrm{m}$ and $12 \mu \mathrm{m}$, if the resolution is sufficient, the HII region PDR appears bright in part because of emission of PAHs. At $24 \mu \mathrm{m}$ and $70 \mu \mathrm{m}$, if the resolution is sufficient, the HII region PDRs are detected with a similar morphology as that seen at $8.0 \mu \mathrm{m}$. There is also, however, IR emission from the region spatially coincident with the ionized gas (see Watson et al. 2008, 2009; Deharveng et al. 2010; Anderson et al. 2011). This emission likely comes from a different dust grain population than the emission in the PDR. It is detected at wavelengths from $12 \mu \mathrm{m}$ to $70 \mu \mathrm{m}$ (see 

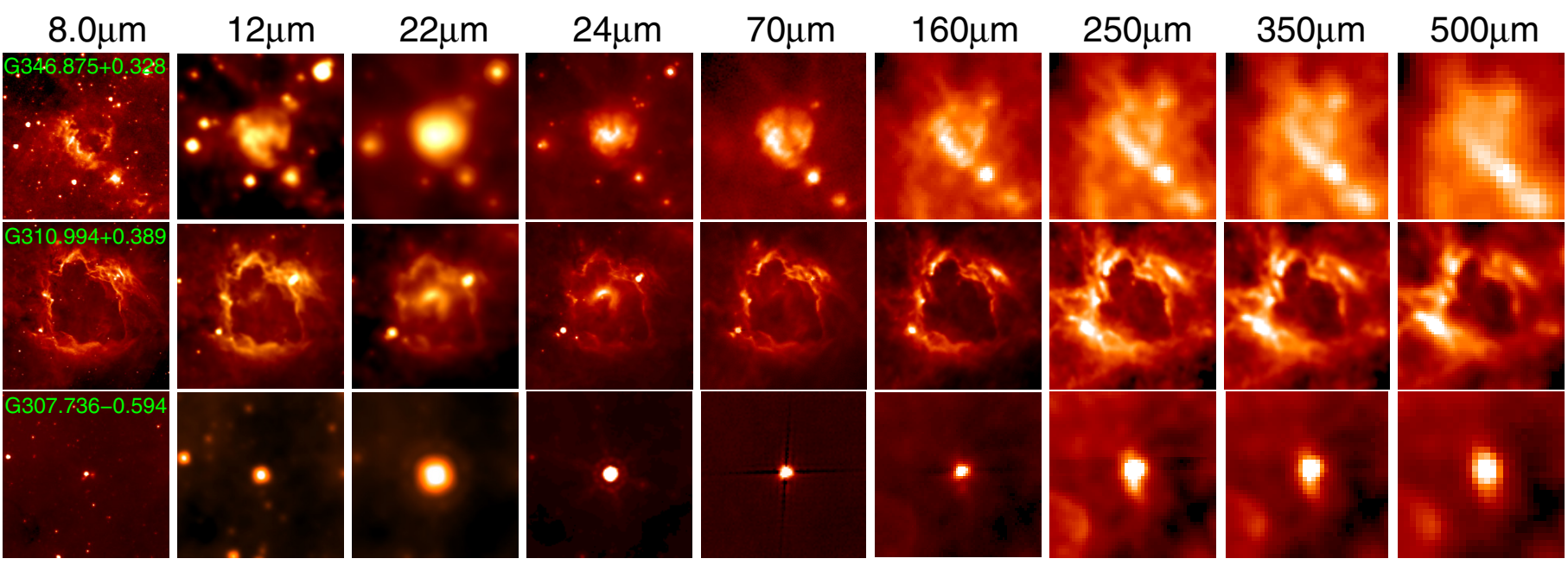

Fig. 1. Infrared images of $\mathrm{H}$ II regions from $8.0 \mu \mathrm{m}$ to $500 \mu \mathrm{m}$ for three representative sources. Shown are G346.875+0.328, G310.994+0.389, and G307.736-0.594 from top to bottom. The images for G346.875+0.328 and G307.736-0.594 are 5' on a side while those of G310.994+0.389 are $12^{\prime}$ on a side.

G310.994+0.389 in Fig. 1). At wavelengths longer than $70 \mu \mathrm{m}$, the dust emission associated with the H II regions here studied is almost entirely from the colder dust in the PDR. This emission again has a similar morphology as the emission at $8.0 \mu \mathrm{m}$, although nearby local filaments associated with the H II regions are also sometimes detected. For angularly small sources, the emission from the H II region is frequently found along a filament whose emission at Herschel SPIRE wavelengths suggests a cold temperature (for example, the emission from a filament with a dust temperature of $15 \mathrm{~K}$ peaks near $200 \mu \mathrm{m}$ and this filament would emit strongly in the $250 \mu \mathrm{m}$ SPIRE band). For these sources, their small angular size and location along a cold filament together hint at an early evolutionary stage.

In Fig. 1, G346.875+0.328 and G310.994+0.389 would not be confused for PNe due to their extended emission. For G307.736-0.594, however, the situation is less clear from a visual inspection alone.

\subsection{Planetary nebulae}

Our goal in creating the PNe sample is to have as many sources as possible, while excluding sources misclassified as $\mathrm{PNe}$. Because we are interested in determining representative FIR colors, we require that all PNe are detected in at least two of the IR bands considered here.

We compile our sample of PNe from Kohoutek (2001), and the Macquarie-AAO-Strasbourg $\mathrm{H} \alpha$ PN Project (MASH) catalogs of more recently discovered PNe (Parker et al. 2006; Miszalski et al. 2008). We also include PNG313.3+00.3 from Cohen et al. (2005). Based on their photometric and spectroscopic observations, the MASH catalogs have a qualitative estimate of whether each source is a "true" PN, or whether it is merely "likely" or "possible". We include only "true" PNe in our sample and exclude MASH sources classified as "likely" or "possible" PNe. We also exclude PNe in complicated zones of the Galaxy where accurate photometry would be difficult. Following Cohen et al. (2011), we remove objects in Kohoutek (2001) whose classification as PNe is questionable. Our sample includes 43 PNe, 25 from Kohoutek (2001), nine from Parker et al. (2006), eight from Miszalski et al. (2008), and one source from Cohen et al. (2005).
Example PNe images are shown in Fig. 2, which shows data from $8.0 \mu \mathrm{m}$ to $500 \mu \mathrm{m}$. While many PNe are resolved at $8.0 \mu \mathrm{m}$, few show extended emission at longer wavelengths. At the longest Herschel wavelengths, the emission from PNe is frequently confused with brighter diffuse features.

Our sample of PNe may not be representative of the entire population of PNe in the Galaxy. Our sample is, however, representative of IR-bright $\mathrm{PNe}$ that may be confused with $\mathrm{H}$ II regions. Many PNe are too faint in the IR to be detected with the suvey data used here. For example, Hora et al. (2008) found that of 233 optically identified PNe in the Large Magellanic Cloud, 161 were detected at $24 \mu \mathrm{m}$ with the MIPS instrument. All sources in our sample are found along the Galactic plane, which may introduce a bias as it will exclude some of the nearest brightest sources that are off the plane.

\section{Aperture photometry}

We perform aperture photometry for all $\mathrm{PNe}$ and $\mathrm{H}$ II regions in our sample using the Kang software ${ }^{4}$, version 1.3. This software treats fractional pixels such that if an aperture encloses $30 \%$ of the area of a pixel, $30 \%$ of the pixel value is included in the aperture flux. Additionally, one can use apertures of arbitrary size and shape and define regions to exclude from the aperture photometry. This flexibility is important for the present analysis in which many regions of interest are in complicated fields, have irregular morphologies, or have nearby contaminating sources of emission.

For the H II regions in our sample, we generally use the same source and background apertures at all wavelengths. When defining apertures we attempt to include all the associated emission at all wavelengths. Most of the $\mathrm{H}$ II regions in our sample are bright at IR wavelengths, are relatively unconfused with nearby sources of emission, and have a similar morphology and angular extent at all wavelengths. For nearly all H II regions, a single source aperture for all wavelengths is sufficient to enclose the associated IR flux from the H II region. We make an exception for some angularly small H II regions and for regions in areas whose background emission varies strongly with wavelength. Very small sources sometimes appear within a small cluster of similar sources at $8.0 \mu \mathrm{m}$, although at longer wavelengths

${ }^{4}$ http://www.bu.edu/iar/kang/ 
L. D. Anderson et al.: Distinguishing $\mathrm{H}$ II regions from PNe
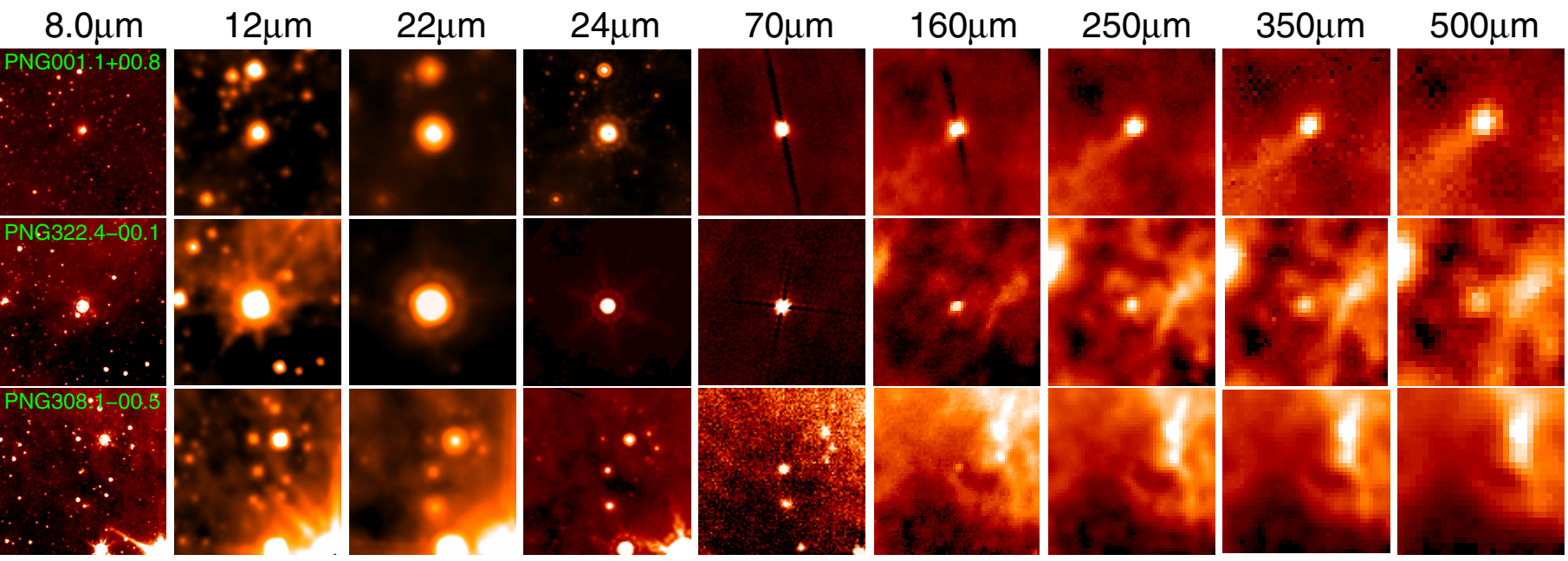

Fig. 2. Infrared images of PNe from $8.0 \mu \mathrm{m}$ to $500 \mu \mathrm{m}$ for three representative sources. Shown are PNG001.1+00.8, PNG322.4-00.1, and PNG308.1-00.5 from top to bottom. All images are 5' on a side. PNG001.1+00.8 and PNG322.4-00.1 are rather bright sources and are detected at all wavelengths, while PNG308.1-00.5 is rather faint and is not detected at wavelengths longer than $160 \mu \mathrm{m}$. The increasing confusion with wavelength is evident.

a single source dominates the emission. An example of this phenomenon is shown in the third row of Fig. 1. For such sources we locate the $8.0 \mu \mathrm{m}$ source that is spatially coincident with the bright source detected at longer wavelengths. We define a small aperture at $8.0 \mu \mathrm{m}$ to isolate the emission from the source responsible for the longer wavelength emission.

We define for each PN separate source and background apertures at each wavelength. Due to their small angular sizes and relatively low IR fluxes, the aperture photometry for the PNe is considerably more sensitive to the aperture size and placement compared to that for H II regions. The choice to define individual apertures at each wavelength was necessitated in part by the background, which varies strongly with wavelength. An appropriate background at $24 \mu \mathrm{m}$ for example may contain large bright diffuse structures at longer wavelengths.

Bright point sources can affect the aperture photometry measurements. For the wavelengths considered here, point source contamination is especially strong from $8.0 \mu \mathrm{m}$ to $24 \mu \mathrm{m}$, where the contaminants are mostly stellar. We exclude the pixel locations of especially bright point sources by hand from the aperture photometry measurements by defining apertures that avoid such features, but we do not remove point sources from the images. The H II regions in our sample are in general much brighter than the combined flux of any point sources within the aperture at the wavelengths considered here; the removal of point sources would have minimal impact on the derived fluxes. Regions of bright extended emission have a dearth of detected point sources because of the increased Poisson $(\sqrt{N})$ noise (see Robitaille et al. 2008, their Fig. 2). Removing the flux from all detected point sources would therefore assign more flux per unit area to the background region compared to the source aperture, leading to an underestimate of the H II region flux. Due to their small angular size, point source contamination is also not generally a problem for PNe since very few point sources are spatially coincident with the PNe themselves. We make no attempt to exclude all point sources from the background apertures, although we do define apertures to exclude bright point sources. Any flux from low-intensity point sources distributed over the background aperture is likely present in the source aperture as well.

We perform aperture photometry using the relation:

$S_{v}=S_{v, 0}-\frac{B_{v}}{N_{B}} \times N_{S}$, where $S_{v}$ is the source flux after the background correction, $S_{v, 0}$ is the flux within the source aperture (before background correction), $B_{v}$ is the flux within the background aperture, $N_{B}$ is the number of pixels in the background aperture, and $N_{S}$ is the number of pixels in the source aperture. This treatment subtracts the mean flux within the background aperture from each pixel in the source aperture. The use of the mean flux in the background aperture rather than the median flux should better estimate the contribution from low-intensity point sources.

For all $\mathrm{H}$ II regions and PNe we define four background apertures and a single source aperture. We use for the measured flux at a given wavelength the average of the four backgroundsubtracted fluxes found after applying Eq. (1) individually to the four source-background pairs. For the uncertainty in flux we use the standard deviation of the four background-subtracted flux measurements.

This method for calculating the flux and uncertainties estimates the true uncertainties associated with our aperture photometry method, which in most cases are dominated by the choice of the background region. In particular, our method accurately estimates errors in fields with strongly varying background emission. In such fields the standard deviation of the pixel values in any given background aperture may be low, but the differences in computed flux between different background apertures may be high.

\section{Results}

The results of the aperture photometry for the $\mathrm{H}$ II regions and $\mathrm{PNe}$ are given in Tables 1 and 2, respectively. Both tables contain the source name followed by the source Galactic longitude and latitude and the angular size (radius) of the aperture used at $24 \mu \mathrm{m}$. For the PNe in Table 2, we list both the Galactic name and the commonly used source identifier. The size column in Table 1 is given in arcmin. while that in Table 2 is given in arcsec. The sizes listed are the "mean radius", calculated from the area of a circular aperture encompassing the same area as the source aperture. Both tables then list in successive columns the flux in Jy and the uncertainties at $8.0 \mu \mathrm{m}, 12 \mu \mathrm{m}, 22 \mu \mathrm{m}, 24 \mu \mathrm{m}$, $70 \mu \mathrm{m}, 160 \mu \mathrm{m}, 250 \mu \mathrm{m}, 350 \mu \mathrm{m}$, and $500 \mu \mathrm{m}$. If more than $0.1 \%$ of the source pixels are saturated at $12 \mu \mathrm{m}, 22 \mu \mathrm{m}$, or $24 \mu \mathrm{m}$ (see 


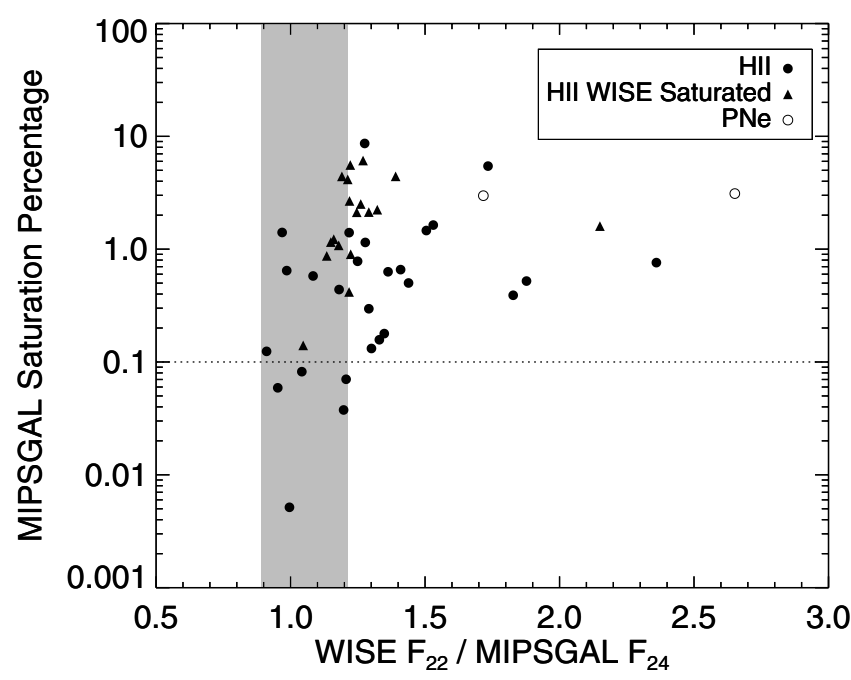

Fig. 3. The ratio of the WISE $22 \mu \mathrm{m}$ flux to the MIPSGAL $24 \mu \mathrm{m}$ flux versus the percentage of MIPSGAL pixels that are saturated. H II regions that have some level of saturation in the WISE $22 \mu \mathrm{m}$ band are shown with open circles and those that do not are shown with filled circles. The two saturated PNe are shown with triangles. The dotted horizonal line shows our cutoff at $0.1 \%$ saturated pixels and the grey area shows one standard deviation of the average of the ratio for all non-saturated $\mathrm{H}$ II regions. The cutoff at $0.1 \%$ separates sources more than one standard deviation from the mean.

below) these fluxes are marked with the symbol: $\dagger$. The final column in both tables is the reference.

If $0.1 \%$ or more the pixels within a source aperture at a given wavelength are saturated such that they have a value of "NAN", we exclude this source from all subsequent analyses involving that wavelength. When there is such severe saturation the aperture photometry fluxes are less reliable. Saturation in the MIPSGAL $24 \mu \mathrm{m}$ data is present for many H II regions and for two PNe in our sample. The WISE data at $12 \mu \mathrm{m}$ and $22 \mu \mathrm{m}$ are also saturated for many sources. In Fig. 3 we show the ratio of the WISE $22 \mu \mathrm{m}$ flux to the MIPSGAL $24 \mu \mathrm{m}$ flux versus the percentage of pixels within the MIPSGAL aperture that are saturated. The average $F_{22}$ to $F_{24}$ ratio for the entire sample of H II regions for which no saturation is detected is $1.05 \pm 0.16$ (see below); this is shown shaded in Fig. 3. The horizontal line at $0.1 \%$ separates sources whose $22 \mu \mathrm{m}$ and $24 \mu \mathrm{m}$ fluxes are more than $25 \%$ different from each other.

Saturation is a problem for many HII regions in the data used here. Forty HII regions have more than $0.1 \%$ of their pixels at $24 \mu \mathrm{m}$ saturated with a value of "NAN": 26 from the Known sample, 12 from the RMS, and two from the HRDS. Additionally, there are two PNe that satisfy this criterion: PNG358.9-00.7 and PNG359.3-00.9. There is also strong saturation in the WISE data, especially at $12 \mu \mathrm{m}$. Thirty H II regions in our sample have more than $0.1 \%$ of their pixels saturated at $12 \mu \mathrm{m}$ (25 from the known sample, four from the RMS and one from the HRDS) and 16 satisfy this criterion at $22 \mu \mathrm{m}$ (15 from the Known sample and one from the RMS). In the WISE bands no PNe in our sample are saturated according to our saturation criterion. PNG359.3-00.9, a PN that shows severe saturation at $24 \mu \mathrm{m}$, however, has an anomalously low flux at $12 \mu \mathrm{m}$ compared to its $8.0 \mu \mathrm{m}$ flux. This indicates that it too may be saturated at $12 \mu \mathrm{m}$; we exclude it from further analysis involving the $12 \mu \mathrm{m}$ flux. Some H II regions show mild saturation at $250 \mu \mathrm{m}$ and $350 \mu \mathrm{m}$, but none meet the $0.1 \%$ cutoff.

\subsection{Detection rates}

At each wavelength we visually assess whether each source was detected. Some sources that we count as non-detections may in fact be marginally detected, but they do not allow the high photometric accuracy required for the present analyses. The detection of a source at a particular wavelength is a function of the intrinsic source brightness, the detector and telescope sensitivity, the manner in which the data were taken, and the local background. Whether a source was detected or not at a particular wavelength therefore does not necessarily reflect the intrinsic properties of a source. On average, however, sources detected at a given wavelength are brighter than those that are not detected at that wavelength.

All H II regions in our sample are detected at all wavelengths. All PNe in our sample are detected at $24 \mu \mathrm{m}$ and $70 \mu \mathrm{m}$. When creating our PNe sample, however, there were four PNe only detected in MIPSGAL that otherwise met our criteria; because we require detection in two wavelength bands, we excluded these sources from our PNe sample. This suggests that the $24 \mu \mathrm{m}$ MIPSGAL band is the most sensitive of the bands studied here for locating PNe, followed by the Hi-GAL $70 \mu \mathrm{m}$ band. Hora et al. (2008) also found that the $24 \mu \mathrm{m}$ band was very sensitive to $\mathrm{PNe}$.

All but one PN is detected in the WISE $22 \mu \mathrm{m}$ data for a $98 \%$ detection rate while three PNe are not detected in the WISE $12 \mu \mathrm{m}$ data for a $93 \%$ detection rate. At $8.0 \mu \mathrm{m}, 91 \%$ of our sample PNe are detected. At wavelengths higher than $70 \mu \mathrm{m}$, the detection percentage steadily decreases: $72 \%$ of the PNe are detected at $160 \mu \mathrm{m}, 49 \%$ at $250 \mu \mathrm{m}, 37 \%$ at $350 \mu \mathrm{m}$, and $28 \%$ at $500 \mu \mathrm{m}$. In part because of the decreasing spatial resolution, as the wavelength increases there is considerable confusion with other sources of emission. This contributes to the decreasing detection rates with increasing wavelength, although the intrinsic faintness of PNe at FIR wavelengths is likely a larger factor.

\subsection{Fluxes}

The H II regions in our sample have significantly higher fluxes compared to the $\mathrm{PNe}$, at all wavelengths. Both samples of objects are composed of relatively bright examples of their classes. The maximum PN flux can be used to set an upper limit that potentially would allow one to separate the two classes.

In Fig. 4, we show the two flux distributions for $\mathrm{H}$ II regions and PNe in the $70 \mu \mathrm{m} \mathrm{Hi-GAL}$ data; the distributions for other photometry bands are similar. For our sample of PNe, the maximum fluxes at $8.0 \mu \mathrm{m}, 12 \mu \mathrm{m}, 22 \mu \mathrm{m}, 24 \mu \mathrm{m}, 70 \mu \mathrm{m}, 160 \mu \mathrm{m}$, $250 \mu \mathrm{m}, 350 \mu \mathrm{m}$, and $500 \mu \mathrm{m}$ are $5.3 \mathrm{Jy}, 6.2 \mathrm{Jy}, 83.2 \mathrm{Jy}, 31.4 \mathrm{Jy}$, 101.6 Jy, $46.0 \mathrm{Jy}, 22.3 \mathrm{Jy}, 7.7 \mathrm{Jy}$, and $3.5 \mathrm{Jy}$, respectively. The maximum $24 \mu \mathrm{m}$ value is saturated and the true value should be closer to the $22 \mu \mathrm{m}$ value. Objects with fluxes higher than these values are unlikely to be PNe, although in extreme cases a bright PNe may have IR fluxes considerably higher than these limits (e.g. IC 418 or NGC 6302).

\subsubsection{A comparison between WISE, MIPS, and IRAS}

The $22 \mu \mathrm{m}$ WISE fluxes are highly correlated with the $24 \mu \mathrm{m}$ MIPSGAL fluxes, as shown in Fig. 5. This is expected due to their similar wavelengths and bandpass shapes. That the WISE and MIPSGAL fluxes are so highly correlated over five orders of magnitude shows that the fidelity of the WISE preliminary release data is adequate for our analyses here. We find that the WISE $22 \mu \mathrm{m}$ band fluxes are on average $1.05 \pm 0.16$ times the 


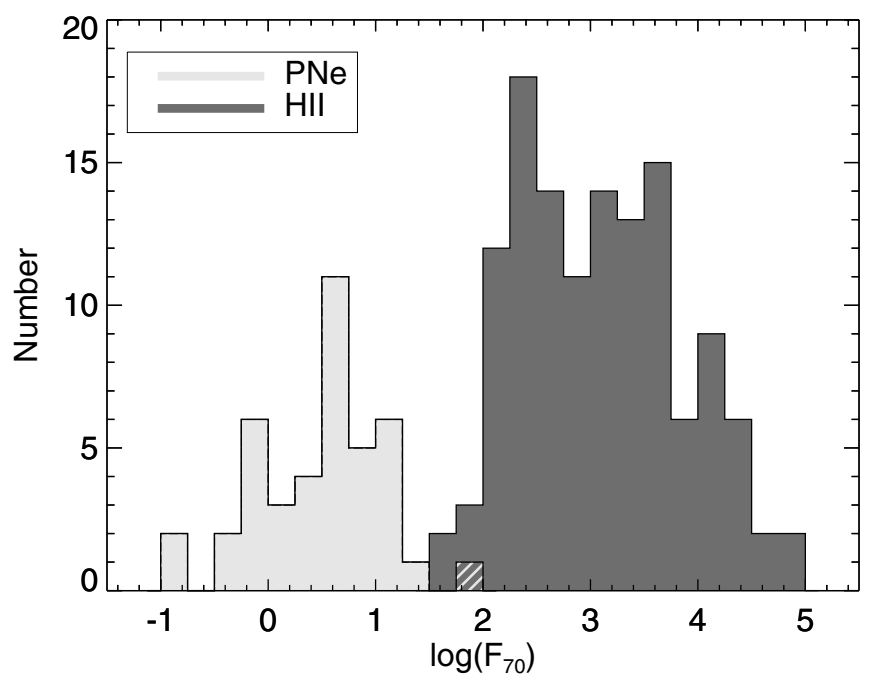

Fig. 4. The distributions of $70 \mu \mathrm{m}$ fluxes. The fluxes of $\mathrm{H}$ II regions are shown in dark grey while those of PNe are shown in light grey. The hatched box has contributions from both $\mathrm{H}$ II regions and PNe. Our sample of $\mathrm{H}$ II regions is significantly brighter than our sample of PNe.

MIPS $24 \mu \mathrm{m}$ fluxes for H II regions that are below our saturation threshold. For PNe, the WISE $22 \mu \mathrm{m}$ band fluxes are on average $1.01 \pm 0.23$ times the MIPS $24 \mu \mathrm{m}$ fluxes. Many of the sources below the line in Fig. 5 are saturated in the $24 \mu \mathrm{m}$ band - we have not excluded saturated sources from the figure.

The $12 \mu \mathrm{m}$ WISE fluxes are less strongly correlated with IRAS $12 \mu \mathrm{m}$ fluxes, as shown in Fig. 6 . To create Fig. 6 we measured the IRAS $12 \mu \mathrm{m}$ fluxes for well-isolated H II regions. We used the same method as before, but we defined new apertures to take into account the significantly poorer IRAS resolution. Contrary to Fig. 5, Fig. 6 shows only sources that are not saturated in the WISE $12 \mu \mathrm{m}$ band according to our saturation criterion. Especially at higher fluxes, we find that the correlation is quite good. Given the larger photometric errors, Fig. 6 is less conclusive than Fig. 5, although it does also suggest that the photometric quality WISE preliminary release data is high.

\subsection{Spectral energy distributions}

Based on the results from IRAS mentioned in the introduction, which showed that $\mathrm{HII}$ regions and PNe possess on average different FIR colors, we expect that the shapes of the spectral energy distributions (SEDs) of $\mathrm{H}$ II regions and PNe are also different. Spectral observations with the Infrared Space Observatory (ISO) have also shown that PNe and $\mathrm{H}$ II regions have different mean SEDs (see Cohen et al. 1999; Peeters et al. 2002b); the SEDs of PNe are generally broader than those of $\mathrm{H}$ II regions.

The average SEDs normalized to the peak flux for PNe and H II regions are shown in Fig. 7. We only include the $12 \mu \mathrm{m}$, $22 \mu \mathrm{m}$, and $24 \mu \mathrm{m}$ fluxes in the averages if the sources are not saturated at these wavelengths according to our saturation criterion. For PNe not detected at all wavelengths, we exclude from the averages the contribution from wavelengths where the source was not detected. The IR emission from PNe evolves with time as the dust in the circumstellar shell expands (e.g García-Lario \& Perea Calderón 2003). Even for the same evolutionary state, whether a given PN is oxygen-rich or carbon-rich can affect its IR properties (Volk \& Kwok 2003; Bernard-Salas 2006). Thus there is significantly more dispersion in the average PN SED

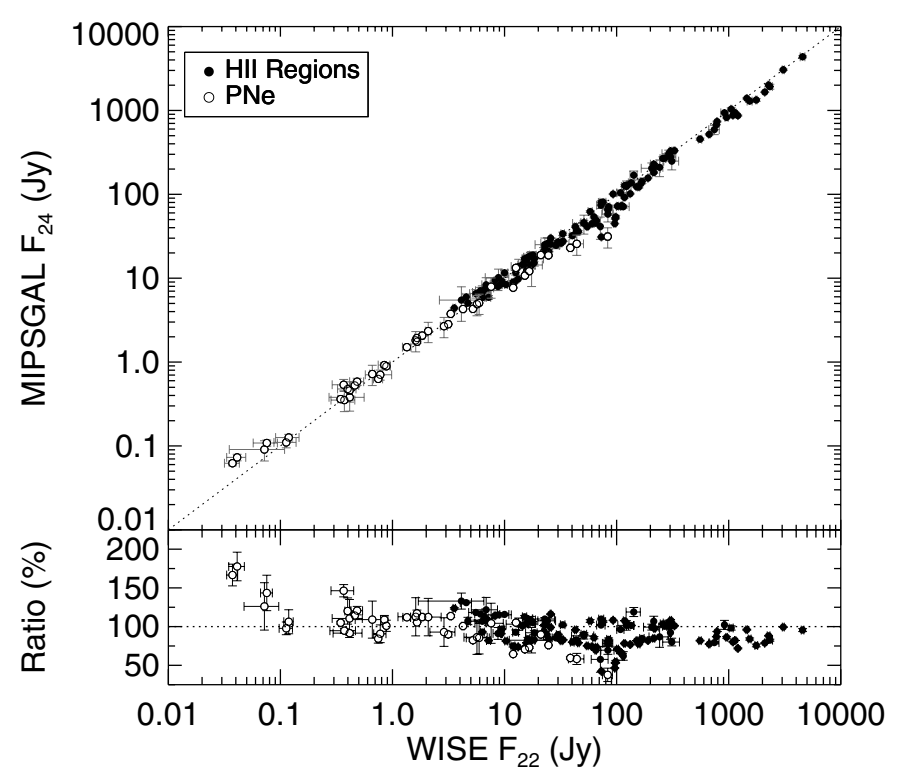

Fig. 5. Correlation between WISE and MIPSGAL. In the top panel we plot the WISE $22 \mu \mathrm{m}$ flux versus the MIPSGAL $24 \mu \mathrm{m}$ flux; in the bottom panel we show the ratio of the MIPSGAL $24 \mu \mathrm{m}$ flux to the WISE $22 \mu \mathrm{m}$ flux, as a percentage. The dotted line in both panels shows a one to one relationship. Errors are the $1 \sigma$ photometric uncertainties. There is a strong correlation between the two fluxes over five orders of magnitude. The sources below the trend line, especially for high values of the flux, indicate MIPSGAL saturation.

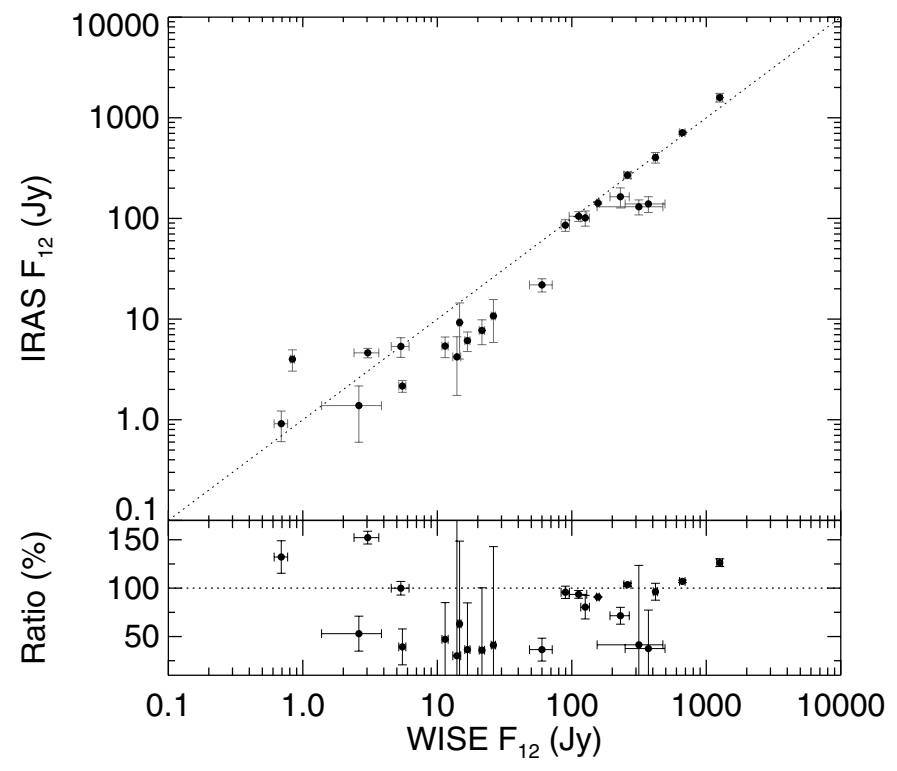

Fig. 6. Correlation between WISE and IRAS. In the top panel we plot the WISE $12 \mu$ m flux versus the IRAS $12 \mu$ m flux; in the bottom panel we show the ratio of the IRAS $12 \mu \mathrm{m}$ flux to the WISE $12 \mu \mathrm{m}$ flux, as a percentage. The dotted line in both panels shows a one to one relationship. Errors are the $1 \sigma$ photometric uncertainties. There is a correlation between the two fluxes over four orders of magnitude, although there is significant scatter in the relationship. This scatter is likely due in large part to the difficulty of IRAS photometric measurements. We have included only $\mathrm{H}$ II regions that are not saturated in the WISE $12 \mu \mathrm{m}$ band.

compared to that of the H II regions, as can be seen in the larger error bars in Fig. 7.

The difference between the average $\mathrm{H}$ II region and $\mathrm{PNe}$ SEDs is clear. For H II regions, the SED peak near $100 \mu \mathrm{m}$ can 


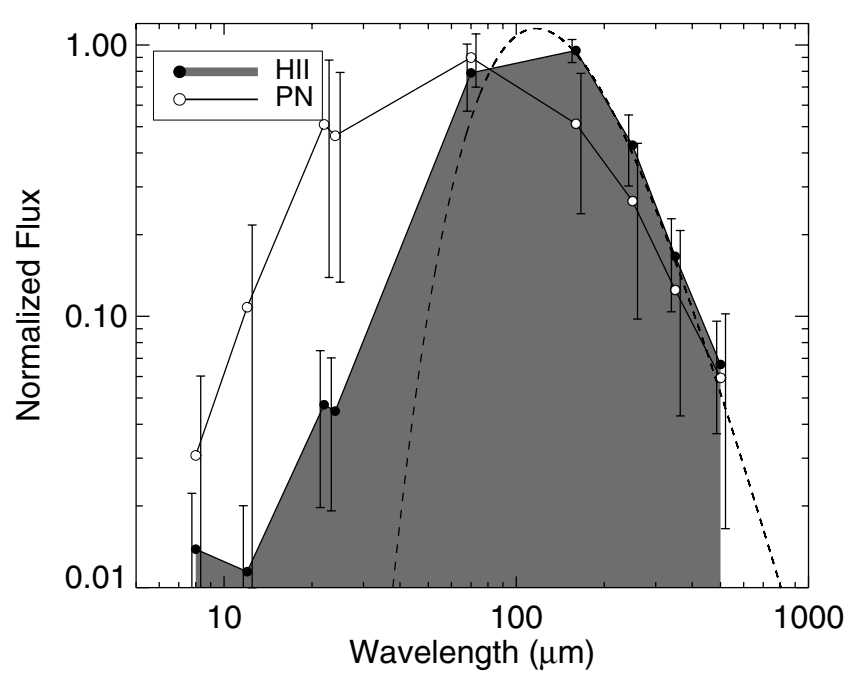

Fig. 7. Spectral energy distributions. Shown are the average normalized spectral energy distributions for $\mathrm{H}$ II regions (dark grey filled area) and PNe (black curve). The SED for dust of temperature $24.8 \mathrm{~K}$ is overplotted with a dashed line. Error bars show the standard deviation in each data point, and are to the left of the points for $\mathrm{H}$ II regions and to the right for PNe. There is considerable difference between the two average SEDs of $\mathrm{H}$ II regions and PNe.

be ascribed to "cold" dust, while in the MIR emission has contributions from warmer dust and emission lines (largely PAHs). The average PN SED rises steeply from $8.0 \mu \mathrm{m}$ to $24 \mu \mathrm{m}$, has a broad peak near $70 \mu \mathrm{m}$, and decreases from $70 \mu \mathrm{m}$ long-ward. We find that a single temperature cannot fit the broad average SED of PNe, indicating that there is dust at a range of temperatures. The average SED for PNe looks similar to what is expected based on the SEDs of individual PNe in Hora et al. (2008) from $8.0 \mu \mathrm{m}$ to $70 \mu \mathrm{m}$. There is, however, considerably less scatter in our $70 \mu \mathrm{m}$ data point than would be expected from their data. Additionally, many of their sources have higher $24 \mu \mathrm{m}$ fluxes than $70 \mu$ m fluxes.

The $12 \mu \mathrm{m}$ WISE flux for H II regions is on average less than the $8.0 \mu \mathrm{m}$ GLIMPSE flux. The average ratio $F_{12} / F_{8}$ is $0.84 \pm$ 0.26 . We do not believe this is a systematic error in the WISE $12 \mu \mathrm{m}$ fluxes because no such trend was found when comparing WISE fluxes with IRAS fluxes (see Fig. 6), but rather is caused by the PAH emission in the $8.0 \mu \mathrm{m}$ band (see also Sect. 5.4).

We find that the "cold" component of the mean H II region SED is well-fit by a single temperature grey body model with a dust temperature $24.8 \pm 0.2 \mathrm{~K}$, when the spectral index of the dust emissivity, $\beta$, is held fixed to a value of 2.0. In these fits, we have assumed $25 \%$ of the emission at $70 \mu \mathrm{m}$ is due to a hotter dust component. Relaxing the assumption at $70 \mu \mathrm{m}$ changes the derived temperatures to $26.3 \mathrm{~K}$, although the fit quality is significantly worse. The mean H II region SED shown in Fig. 7 is very similar to the average SED for "bubble" H II regions shown in Anderson (2011), as are the derived dust temperature values.

Over two-thirds (68\%) of H II regions in our sample peak at $160 \mu \mathrm{m}$, while one third (32\%) peak at $70 \mu \mathrm{m}$. Of the PNe SEDs, $67 \%$ peak at $70 \mu \mathrm{m}, 23 \%$ peak at either $22 \mu \mathrm{m}$ or $24 \mu \mathrm{m}$, and $9 \%$ (two sources) peak at $160 \mu \mathrm{m}$. The two PNe that peak at $160 \mu \mathrm{m}$ are PNG002.2+00.5 and PNG358.6+00.7.

\subsection{Discriminating colors}

We examined the distributions of all possible colors to search for the most robust criteria for discriminating between $\mathrm{H}$ II regions and PNe. The distributions for the most robust colors are shown in Fig. 8. There, the hatched areas contain contributions from both H II regions and PNe. The vertical dotted lines in Fig. 8 are our proposed discriminating color values. Throughout, we use the notation such that flux ratios enclosed in square brackets represent the log of the flux ratio.

In Table 3, we list statistics on the colors shown in Fig. 8, as well the statistics of some additional colors. In Fig. 8, we show colors using the WISE $22 \mu \mathrm{m}$ data in lieu of the MIPSGAL $24 \mu \mathrm{m}$ data, but we list statistics on colors found using both data sets in Table 3. Table 3 gives the color, the proposed discriminating color cutoff value, the percentage of $\mathrm{H}$ II regions and $\mathrm{PNe}$ satisfying this color cutoff, the average value of this color for the $\mathrm{H}$ II regions and $\mathrm{PNe}$ in our sample, together with their standard deviations, and the number of $\mathrm{H}$ II regions and $\mathrm{PNe}$ that were used in the calculations. Table 3 shows that there are many different colors that can robustly separate $\mathrm{PNe}$ and $\mathrm{H}$ II regions. The three most effective colors are $\left[F_{12} / F_{8}\right]<0.3$, which is satisfied by $98 \%$ of $\mathrm{H}$ II regions and $7 \%$ of PNe, $\left[F_{160} / F_{12}\right]>1.3$, which is satisfied by $100 \%$ of $\mathrm{H}$ II regions and $10 \%$ of $\mathrm{PNe}$, and $\left[F_{160} / F_{24}\right]>0.8$, which is also satisfied by $100 \%$ of H II regions and $10 \%$ of PNe $\left(\left[F_{160} / F_{22}\right]>0.8\right.$ is satisfied by $100 \%$ of $\mathrm{H} \mathrm{II}$ regions and $12 \%$ of $\mathrm{PNe})$.

Table 3 shows that $\mathrm{H}$ II regions possess a relatively narrow range of colors. Since our sample has H II regions in a range of evolutionary states, any evolutionary effects in these colors must be relatively modest. The colors of PNe span a broader range of values compared to those of H II regions, for all colors shown in Table 3.

Most of the variation in PNe colors is real and not the result of photometric uncertainties. For example, the standard deviation in $\left[F_{160} / F_{24}\right]$ is 0.55 dex for PNe. Restricting the calculation to the $20 \mathrm{PNe}$ with the highest $160 \mu \mathrm{m}$ flux for which the photometric uncertainty is relatively low, the standard deviation is 0.50 ; it is 0.52 for the 10 brightest PNe. Similarly, the $\left[F_{70} / F_{24}\right]$ color has a standard deviation of 0.47 for $\mathrm{PNe}$; this standard deviation is 0.43 and 0.38 for the 20 and 10 brightest $\mathrm{PNe}$ at $70 \mu \mathrm{m}$, respectively.

The colors that we have found most useful for discriminating between $\mathrm{H}$ II regions and $\mathrm{PNe}$ can be divided into three categories. First, many useful colors compare the flux at $70 \mu \mathrm{m}$ or $160 \mu \mathrm{m}$ with the flux in the MIR at $12 \mu \mathrm{m}, 22 \mu \mathrm{m}$, or $24 \mu \mathrm{m}$. A simple interpretation of these colors is that they compare the emission from cold dust to that of a warmer dust component. Figure 7 shows that the average PN SED has significantly more emission in the MIR. This suggests that, relative to the cold dust component, PNe have more emission from warm dust than do H II regions. The second category are the colors comparing $12 \mu \mathrm{m}, 22 \mu \mathrm{m}$, or $24 \mu \mathrm{m}$ fluxes with $8 \mu \mathrm{m}$ fluxes. This ratio is sensitive to the emission from PAHs, which emit strongly at $8.0 \mu \mathrm{m}$, and less strongly in the other bands. As stated earlier, PAHs emit much more strongly for $\mathrm{H}$ II regions than for PNe. Finally, the ratio $\left[F_{160} / F_{70}\right]$ is sensitive to the location of the SED peak. Since on average the SEDs of H II regions peak at longer wavelengths than PNe, this ratio will be positive for most H II regions and negative for most PNe.

\subsection{Color-color diagrams}

There are numerous color combinations where H II regions and $\mathrm{PNe}$ are strongly segregated; the utility of an individual colorcolor plot depends on the available measurements and their accuracy. We show in Fig. 9 color-color diagrams for two of the most robust discriminating colors. The left panel of Fig. 9 shows the 
L. D. Anderson et al.: Distinguishing H II regions from PNe
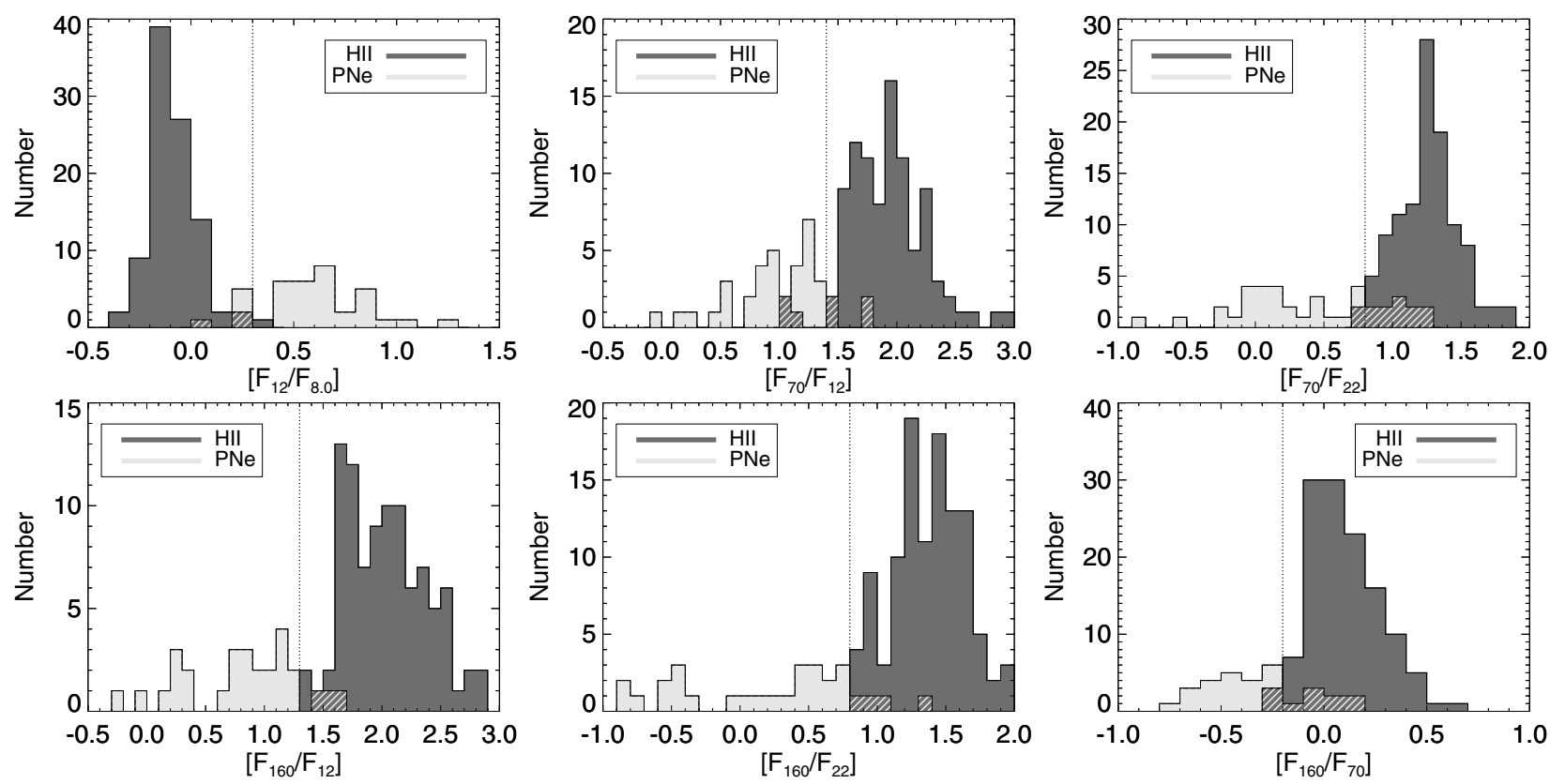

Fig. 8. IR color distributions for $\mathrm{H}$ II regions and PNe. Shown are the six colors (the log of the flux ratio) best able to discriminate between $\mathrm{H}$ II regions (dark grey) and PNe (light grey). The hatched areas contain contributions from both H II regions and PNe. The vertical dotted lines show our proposed cutoff value for separating the two populations.

Table 3. IR colors for disinguishing H II regions from PNe.

\begin{tabular}{lrrrrrrr}
\hline \hline Color & Cutoff & $\%$ H II & $\%$ PNe & Avg. (HII) & Avg. (PNe) & \#H II & \#PNe \\
\hline$\left[F_{12} / F_{8}\right]$ & $<0.3$ & 98 & 18 & $-0.09 \pm 0.11$ & $0.57 \pm 0.29$ & 96 & 38 \\
{$\left[F_{22} / F_{8}\right]$} & $<1.0$ & 92 & 20 & $0.59 \pm 0.31$ & $1.23 \pm 0.34$ & 110 & 39 \\
{$\left[F_{24} / F_{8}\right]$} & $<1.0$ & 93 & 21 & $0.55 \pm 0.29$ & $1.23 \pm 0.31$ & 86 & 37 \\
{$\left[F_{70} / F_{12}\right]$} & $>1.3$ & 96 & 20 & $1.92 \pm 0.36$ & $1.01 \pm 0.40$ & 96 & 40 \\
{$\left[F_{70} / F_{22}\right]$} & $>0.8$ & 98 & 26 & $1.25 \pm 0.22$ & $0.39 \pm 0.51$ & 110 & 42 \\
{$\left[F_{70} / F_{24}\right]$} & $>0.8$ & 100 & 26 & $1.26 \pm 0.21$ & $0.42 \pm 0.47$ & 86 & 41 \\
{$\left[F_{160} / F_{12}\right]$} & $>1.3$ & 100 & 10 & $2.06 \pm 0.38$ & $0.80 \pm 0.49$ & 96 & 30 \\
{$\left[F_{160} / F_{22}\right]$} & $>0.8$ & 100 & 12 & $1.38 \pm 0.26$ & $0.16 \pm 0.64$ & 110 & 31 \\
{$\left[F_{160} / F_{24}\right]$} & $>0.8$ & 100 & 10 & $1.40 \pm 0.25$ & $0.22 \pm 0.55$ & 86 & 29 \\
{$\left[F_{160} / F_{70}\right]$} & $>-0.2$ & 97 & 25 & $0.10 \pm 0.17$ & $-0.32 \pm 0.24$ & 126 & 31 \\
\hline
\end{tabular}

colors $\left[F_{12} / F_{8}\right]$ versus $\left[F_{160} / F_{22}\right]$ while the right panel shows $\left[F_{70} / F_{22}\right]$ versus $\left[F_{160} / F_{12}\right]$. The black dashed lines in each panel enclose $100 \%$ of our sample H II regions. In the left panel, the area enclosed by these dashed lines includes no PNe while in the right panel, only two PNe are within the area. The combination of two colors therefore shows a modest improvement in discriminating power compared to the single colors alone.

\section{Application to objects of unknown classification}

There are numerous astrophysical objects that the criteria here derived can hopefully help classify. The power of these criteria is of course limited to separating $\mathrm{HII}$ regions from PNe, and may not be applicable if the object in question is neither an H II region nor a PN. Below, we test our derived criteria on additional samples of objects of unknown or contested classification. We also test the criteria on a sample of known PNe that are outside the coverage of Hi-GAL.

Some of the objects listed as PNe in Kwok et al. (2008) are not included in other catalogs of PNe. Most such objects appear visually similar to $\mathrm{H}$ II regions: they have diffuse MIR emission extended over multiple arcminutes and show poor symmetry. We perform aperture photometry from $8.0 \mu \mathrm{m}$ to $500 \mu \mathrm{m}$ as before (see Sect. 4) on seven such nebulae that fall within the Galactic zone studied here. These seven nebulae are listed in Kwok et al. (2008) as PNG298.4+00.6, PNG301.2+00.4, PNG321.0-00.7, PNG329.6-00.4, PNG332.5-00.1, PNG333.7+00.3, and PNG340.0+00.9. We find that six of these sources satisfy all the criteria listed in Table 3 for H II regions; their IR colors support their assignment as $\mathrm{HII}$ regions rather than PNe. PNG321.0-00.7, however, has colors more similar to PNe. For example, its ratio $\left[F_{160} / F_{12}\right]$ is 0.65 . This source is only a few arcseconds in diameter and seems likely to be a PN.

Many of the "disk and ring" sources seen in MIPSGAL that were cataloged by Mizuno et al. (2010) do not have definitive classifications. Mizuno et al. (2010) make a strong case that the catalog consists primarily of evolved stars, and therefore we would expect few HII regions. They list in their Appendix, however, two objects that based on their morphologies may be previously unclassified spiral galaxies. One of these, MGE351.2381-00.0145, shown in their Fig. 12, appears visually similar to an HII region. For this object, the $24 \mu \mathrm{m}$ emission is surrounded by an $8.0 \mu \mathrm{m}$ ring. The morphology of MGE351.2381-00.0145 is similar to the HII region G034.325+0.211 detected in the $\mathrm{HRDS}^{5}$. The IR colors

\footnotetext{
${ }^{5}$ See http://www.cv.nrao.edu/hrds/three_color/three_ color_LA229.jpg
} 

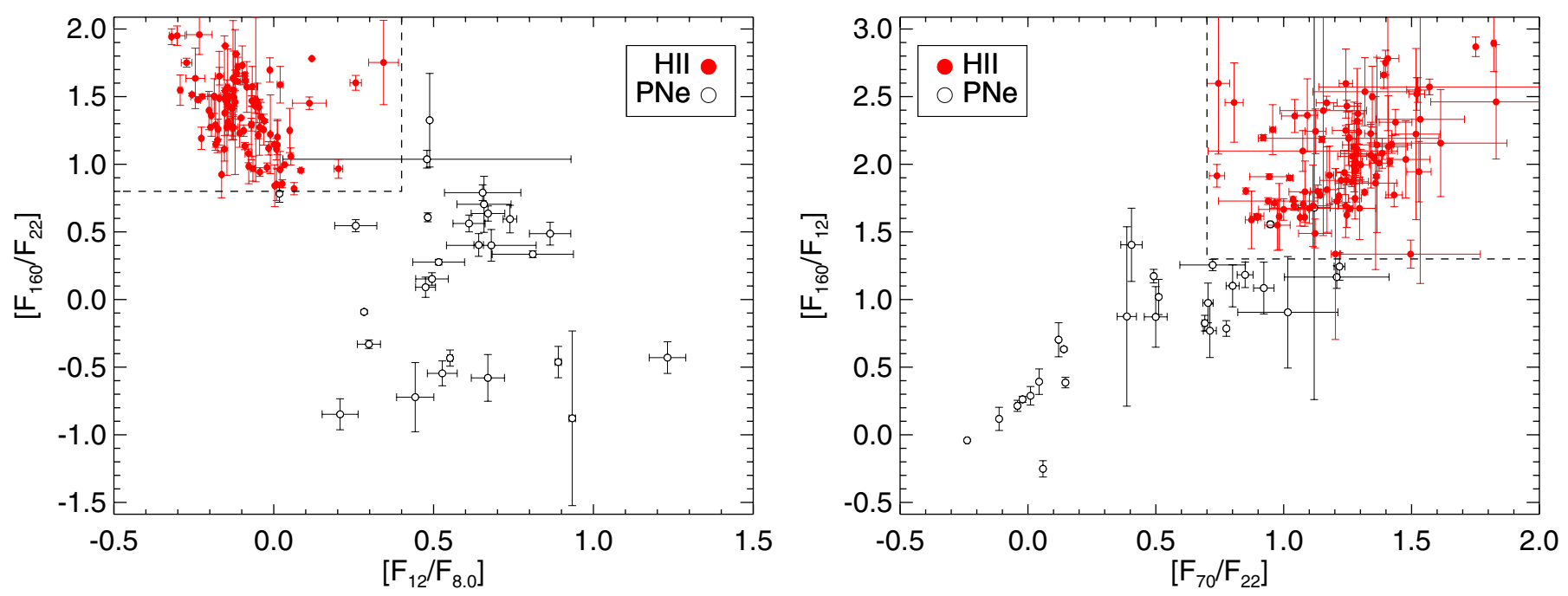

Fig. 9. Color-color diagrams for $\mathrm{H}$ II regions and PNe. The left panel shows the colors $\left[F_{12} / F_{8}\right]$ versus $\left[F_{160} / F_{22}\right]$ while the right panel shows $\left[F_{70} / F_{22}\right]$ versus $\left[F_{160} / F_{12}\right]$. The dashed lines in both panels enclose all $\mathrm{H}$ II regions and few PNe: none in the left panel and two in the right panel.

of MGE351.2381-00.0145 are consistant with it being an H II region: its ratio $\left[F_{12} / F_{8}\right]$ is -0.42 , and $\left[F_{22} / F_{8}\right]$ is -0.39 . We propose that this is an HII region, and the "spiral" geometry arises from line-of-sight effects, as it likely also does for G034.325+0.211. An additional object, named MGE031.7290+00.6993 in Mizuno et al. (2010), is also included in the HRDS as G031.727+0.698. This object also has $24 \mu \mathrm{m}$ emission surrounded by $8.0 \mu \mathrm{m}$ emission ${ }^{6}$. Its ratio $\left[F_{12} / F_{8}\right]$ is -0.58 , and $\left[F_{22} / F_{8}\right]$ is -0.13 , consistant with it being an H II region. Neither object is included in the Hi-GAL data range here studied ${ }^{7}$.

There are 14 HRDS sources that appear point-like at $8.0 \mu \mathrm{m}$. Based on their morphology, they are likely PNe rather than $\mathrm{H}$ II regions, although the line widths of their recombination line emission suggest that what was detected does not arise from $\mathrm{PNe}$ (see Anderson et al. 2011). None of these sources lie within the Hi-GAL range studied here. Of these 14 sources, only three satisfy $\left[F_{12} / F_{8}\right]>0.3$, and eight satisfy $\left[F_{22} / F_{8}\right]>1.0$, the colors associated with PNe. Based on these colors, some of these sources many in fact be H II regions. Without Hi-GAL data, however, our discriminating power is more limited (see Table 3).

Finally, there are a number of PNe near the Galactic Center region that have Spitzer data but, because of their Galactic latitudes, do not have Hi-GAL data. We analyze 11 such objects, using only GLIMPSE, MIPSGAL, and WISE data. We find that of these 11 , seven satisfy $\left[F_{12} / F_{8}\right]>0.3$ and $\left[F_{22} / F_{8}\right]>1.0$, consistant with the colors derived for $\mathrm{PNe}$.

The low success rates above show the importance of Hi-GAL data for such studies. The central white dwarf of PNe can be detected at MIR wavelengths. The contribution from the central source, relative to any contribution from a dusty shell, increases with decreasing wavelength. We hypothesize that the flux from the central source decreases the discriminatory power of IR colors at lower wavelengths.

\footnotetext{
${ }^{6}$ See http://wwW.cv.nrao.edu/hrds/three_color/three_ color_LA014.jpg

7 Between submission and publication we analyzed additional HiGAL data that were not originally available. Both sources satisfy all the criteria for $\mathrm{H}$ II regions in Table 3.
}

\section{Summary}

Using data from Herschel Hi-GAL, WISE, Spitzer MIPSGAL, and Spitzer GLIMPSE, we have searched for the most robust colors for separating a sample of $126 \mathrm{H}$ II regions from a sample of 43 planetary nebulae (PNe). While our sample of $\mathrm{PNe}$, which is confined to the Galactic plane and infrared bright, may not be representative of the entire Galactic population, it should be representative of $\mathrm{PNe}$ that may be confused with $\mathrm{H}$ II regions.

$\mathrm{H}$ II regions and PNe detected at MIR and FIR wavelengths can be reliably separated using these data, and numerous colors can be used to do so. The best color criteria are $\left[F_{12} / F_{8}\right]<0.3$, which is satisfied by $98 \%$ of our sample of $\mathrm{H}$ II regions and $7 \%$ of our sample of PNe, $\left[F_{160} / F_{12}\right]>1.3$, which is satisfied by $100 \%$ of our sample of $\mathrm{H}$ II regions and $10 \%$ of our sample of PNe, and $\left[F_{160} / F_{24}\right]>0.8$ which is also satisfied by $100 \%$ of our sample of $\mathrm{H}$ II regions and $10 \%$ of our sample of PNe. Long wavelength Hi-GAL data greatly increase the discrimatory power of such IR color criteria. We applied these criteria to objects of unknown classification and were able to discriminate between $\mathrm{H}$ II regions and PNe in most cases. Replacing the MIPSGAL $24 \mu \mathrm{m}$ fluxes with those of the WISE $22 \mu \mathrm{m}$ band in the previous ratio produces a nearly identical result. The colors of H II regions span a relatively narrow range of values. Any evolution in these H II region colors with age must therefore be relatively small.

Combinations of these colors can be more effective at separating $\mathrm{HII}$ regions and PNe. For example the combination $\left[F_{12} / F_{8}\right]<0.4$ and $\left[F_{160} / F_{22}\right]>0.8$ includes all $\mathrm{H}$ II regions in our sample and no PNe.

The average SEDs of the $\mathrm{H}$ II regions and $\mathrm{PNe}$ in our sample are significantly different. The average SED of $\mathrm{H}$ II regions has an obvious "cold" component that peaks near $100 \mu \mathrm{m}$, as well as warmer component(s) that emit in the MIR and a strong contribution at $8.0 \mu \mathrm{m}$ that may be caused by PAHs. The average SED of PNe is much broader than that of $\mathrm{H}$ II regions and from these data cannot be separated cleanly into temperature components. The "cold" component of the H II region SED is well-fit with a grey-body of dust temperature $25 \mathrm{~K}$. The SED of over twothirds of H II regions peaks at $160 \mu \mathrm{m}$, while the remaining one third peak at $70 \mu \mathrm{m}$. The SEDs of $67 \%$ of PNe peak at $70 \mu \mathrm{m}$, 
with the remainder peaking at either $22 \mu \mathrm{m}$ or $24 \mu \mathrm{m}(23 \%)$ or $160 \mu \mathrm{m}(9 \%)$.

We find that WISE $22 \mu \mathrm{m}$ fluxes are strongly correlated with MIPSGAL $24 \mu \mathrm{m}$ fluxes over five orders of magnitude. This suggests that the WISE preliminary release data have sufficient accuracy for the photometric measurements carried out here. The WISE $12 \mu \mathrm{m}$ data are also well-correlated with the IRAS $12 \mu \mathrm{m}$ data, although the scatter is larger in part due to the larger uncertainties when performing aperture photometry with IRAS data.

These results are important for future work on H II regions and PNe since the two populations can be reliably separated using photometric measurements and the discriminating IR colors derived here. These criteria will be useful when defining samples of $\mathrm{H}$ II regions and $\mathrm{PNe}$ from large-angle sky surveys.

Acknowledgements. We would like to thank the Hi-GAL team for their continuing work on the survey. PACS has been developed by a consortium of institutes led by MPE (Germany) and including UVIE (Austria); KU Leuven, CSL, IMEC (Belgium); CEA, LAM (France); MPIA (Germany); INAF-IFSI/OAA/OAP/OAT, LENS, SISSA (Italy); IAC (Spain). This development has been supported by the funding agencies BMVIT (Austria), ESAPRODEX (Belgium), CEA/CNES (France), DLR (Germany), ASI/INAF (Italy), and CICYT/MCYT (Spain). SPIRE has been developed by a consortium of institutes led by Cardiff University (UK) and including Univ. Lethbridge (Canada); NAOC (China); CEA, LAM (France); IFSI, Univ. Padua (Italy); IAC (Spain); Stockholm Observatory (Sweden); Imperial College London, RAL, UCL-MSSL, UKATC, Univ. Sussex (UK); and Caltech, JPL, NHSC, Univ. Colorado (USA). This development has been supported by national funding agencies: CSA (Canada); NAOC (China); CEA, CNES, CNRS (France); ASI (Italy); MCINN (Spain); SNSB (Sweden); STFC (UK); and NASA (USA). This publication makes use of data products from the Wide-field Infrared Survey Explorer, which is a joint project of the University of California, Los Angeles, and the Jet Propulsion Laboratory/California Institute of Technology, funded by the National Aeronautics and Space Administration. This research has made use of NASA's Astrophysics Data System Bibliographic Services and the SIMBAD database operated at CDS, Strasbourg, France. L.D.A. acknowledges support by the ANR Agence Nationale for the research project "PROBeS", number ANR08-BLAN-0241.

\section{References}

Aaquist, O. B., \& Kwok, S. 1990, A\&AS, 84, 229

Anderson, L. D. 2011, A\&A, submitted

Anderson, L. D., Zavagno, A., Rodón, J. A., et al. 2010, A\&A, 518, L99

Anderson, L. D., Bania, T. M., Balser, D. S., \& Rood, R. T. 2011, ApJS, 194, 32

Bania, T. M., Anderson, L. D., Balser, D. S., \& Rood, R. T. 2010, ApJ, 718, L106

Benjamin, R. A., Churchwell, E., Babler, B. L., et al. 2003, PASP, 115, 953

Bernard-Salas, J. 2006, in Planetary Nebulae in our Galaxy and Beyond, ed. M. J. Barlow, \& R. H. Méndez, IAU Symp., 234, 181

Carey, S. J., Mizuno, D. R., Kraemer, K. E., et al. 2008, MIPSGAL v3.0 Data Delivery Description Document (29 August 2008)

Carey, S. J., Noriega-Crespo, A., Mizuno, D. R., et al. 2009, PASP, 121, 76

Caswell, J. L., \& Haynes, R. F. 1987, A\&A, 171, 261

Chu, Y., Gruendl, R. A., Guerrero, M. A., et al. 2009, AJ, 138, 691
Churchwell, E., Babler, B. L., Meade, M. R., et al. 2009, PASP, 121, 213 Cohen, M. 2009, AJ, 137, 3449

Cohen, M., Barlow, M. J., Sylvester, R. J., et al. 1999, ApJ, 513, L135

Cohen, M., Green, A. J., Roberts, M. S. E., et al. 2005, ApJ, 627, 446

Cohen, M., Parker, Q. A., Green, A. J., et al. 2007, ApJ, 669, 343

Cohen, M., Parker, Q. A., Green, A. J., et al. 2011, MNRAS, 413, 514

Condon, J. J., \& Kaplan, D. L. 1998, ApJS, 117, 361

Deharveng, L., Schuller, F., Anderson, L. D., et al. 2010, A\&A, 523, A6

Egan, M. P., Price, S. D., Kraemer, K. E., et al. 2003, Air Force Research Laboratory Technical Report AFRL-VS-TR-2003-1589

Fazio, G. G., Hora, J. L., Allen, L. E., et al. 2004, ApJS, 154, 10

García-Lario, P., \& Perea Calderón, J. V. 2003, in Exploiting the ISO Data Archive, Infrared Astronomy in the Internet Age, ed. C. Gry, S. Peschke, J. Matagne, P. Garcia-Lario, R. Lorente, \& A. Salama, ESA Spec. Publ., 511, 97

Griffin, M. J., Abergel, A., Abreu, A., et al. 2010, A\&A, 518, L3

Hora, J. L., Latter, W. B., Allen, L. E., et al. 2004, ApJS, 154, 296

Hora, J. L., Cohen, M., Ellis, R. G., et al. 2008, AJ, 135, 726

Hughes, V. A., \& MacLeod, G. C. 1989, AJ, 97, 786

Kohoutek, L. 2001, A\&A, 378, 843

Kwok, S., Zhang, Y., Koning, N., Huang, H., \& Churchwell, E. 2008, ApJS, 174, 426

Lockman, F. J. 1989, ApJS, 71, 469

Lockman, F. J., Pisano, D. J., \& Howard, G. J. 1996, ApJ, 472, 173

Milne, D. K., \& Aller, L. H. 1975, A\&A, 38, 183

Miszalski, B., Parker, Q. A., Acker, A., et al. 2008, MNRAS, 384, 525

Miville-Deschênes, M.-A., \& Lagache, G. 2005, ApJS, 157, 302

Mizuno, D. R., Kraemer, K. E., Flagey, N., et al. 2010, AJ, 139, 1542

Molinari, S., Swinyard, B., Bally, J., et al. 2010, A\&A, 518, L100

Parker, Q. A., Acker, A., Frew, D. J., et al. 2006, MNRAS, 373, 79

Peeters, E., Hony, S., Van Kerckhoven, C., et al. 2002a, A\&A, 390, 1089

Peeters, E., Martín-Hernández, N. L., Damour, F., et al. 2002b, A\&A, 381, 571

Phillips, J. P., \& Marquez-Lugo, R. A. 2011, MNRAS, 410, 2257

Phillips, J. P., \& Ramos-Larios, G. 2008, MNRAS, 391, 1527

Poglitsch, A., Waelkens, C., Geis, N., et al. 2010, A\&A, 518, L2

Pottasch, S. R., Olling, R., Bignell, C., \& Zijlstra, A. A. 1988, A\&A, 205, 248

Price, S. D., Egan, M. P., Carey, S. J., Mizuno, D. R., \& Kuchar, T. A. 2001, AJ, 121,2819

Rieke, G. H., Young, E. T., Engelbracht, C. W., et al. 2004, ApJS, 154, 25

Robitaille, T. P., Meade, M. R., Babler, B. L., et al. 2008, AJ, 136, 2413

Rodón, J. A., Zavagno, A., Baluteau, J., et al. 2010, A\&A, 518, L80

Sewilo, M., Churchwell, E., Kurtz, S., Goss, W. M., \& Hofner, P. 2004, ApJ, 605,285

Tielens, A. G. G. M. 2008, ARA\&A, 46, 289

Urquhart, J. S., Busfield, A. L., Hoare, M. G., et al. 2007a, A\&A, 461, 11

Urquhart, J. S., Busfield, A. L., Hoare, M. G., et al. 2007b, A\&A, 474, 891

Urquhart, J. S., Hoare, M. G., Lumsden, S. L., Oudmaijer, R. D., \& Moore, T. J. T. 2008, in Massive Star Formation: Observations Confront Theory, ed. H. Beuther, H. Linz, \& T. Henning, ASP Conf. Ser., 387, 381

Volk, K., \& Kwok, S. 2003, in Planetary Nebulae: Their Evolution and Role in the Universe, ed. S. Kwok, M. Dopita, \& R. Sutherland, IAU Symp., 209, 303

Watson, C., Povich, M. S., Churchwell, E. B., et al. 2008, ApJ, 681, 1341

Watson, C., Corn, T., Churchwell, E. B., et al. 2009, ApJ, 694, 546

Westerhout, G. 1958, Bull. Astron. Inst. Netherlands, 14, 215

Wood, D. O. S., \& Churchwell, E. 1989, ApJ, 340, 265

Wright, E. L., Eisenhardt, P. R. M., Mainzer, A. K., 2010, AJ, 140, 1868

Zhang, Y., \& Kwok, S. 2009, ApJ, 706, 252

Zijlstra, A. A., Pottasch, S. R., \& Bignell, C. 1989, A\&AS, 79, 329 